\title{
Thermal base drive for micromechanical resonators employing deep-diffusion bases
}

\author{
Siebe Bouwstra*, Jeroen van Rooijen and Harrie A C T1mans \\ MESA Research Unit, University of Twente, PO Box 217, 7500 AE Enschede (Netherlands)
}

Arjun Selvakumar and Khalıl Najafi
Center for Integrated Sensors and Circutts, University of Michigan, 1301 Beal Ave, Ann Arbor, MI 48109-2122 (USA)

\begin{abstract}
A novel approach of thermal excitation is presented, where thin micromechancal structures are suspended by deep-diffusion bases Cantilevers and microbridges are fabricated, modeled and tested Resonance frequencies are solely determined by the thin parts of the structures, and are independent of matenal properties and dimensions of the base The efficiency for the amplitude of vibration is independent of the thickness and length of the base Therefore short and thick bases can be applied, leading to relatively small temperature elevations inherent to thermal excitation
\end{abstract}

\section{Introduction}

Micromechanical resonators have a wide application field for sensors as well as for actuators [1-3] Up till now, two approaches have been applied for excitation and detection of the vibrations One makes use of elements external to the resonator, exerting body or surface loads for excitation, and directly sensing displacements for detection Examples are the electrostatic excitation and capacitive detection [4-7], and the Lorentz force excitation and inductive detection $[8,9]$

A second approach makes use of elements integrated within the resonator In this approach excitation of the mechanical vibration is achieved by forced deformation of the excitation element, and detection is obtained from the deformation of the detection element Examples are piezoelectric excitation and detection [10-12], and thermal excitation and piezoresistive detection [1316] Neither of these approaches is ideal A disadvantage of the approach with external elements is that a narrow air gap is required This leads to limited room to move and to a large viscous damping due to squeezing of the alr film, necessitating vacuum conditions Also the gap puts requirements on the fabrication technology, and on the shape of the structure In the approach with internal elements the materials of the driving elements are usually not optımızed for mechan-

*Present address MIC, Technıcal University of Denmark, DK2800 Lyngby, Denmark ical properties, but instead for electrical and electromechanical transduction properties Also, differential initial strains between these materials lead to initial bending and wrinkling of the structure Furthermore, the initial strains can depend on operational conditions, such as the static temperature elevation in the case of thermal excitation [17]

A novel approach to the excitation and detection of the mechanical vibrations of micromechanical structures, called 'base drive', was presented in ref 18 In this approach the driving elements are embedded in the base or support of the mucromechanical structure, while the compliant part of the structure is of a simple geometry, and consists of a single material only Different configurations were investigated Figure 1 shows the example of a bending base drive with thermal excitation, where the base is realized by deep boron diffusion and $\mathrm{p}^{++}$-etch stop [19] When the base is forced to

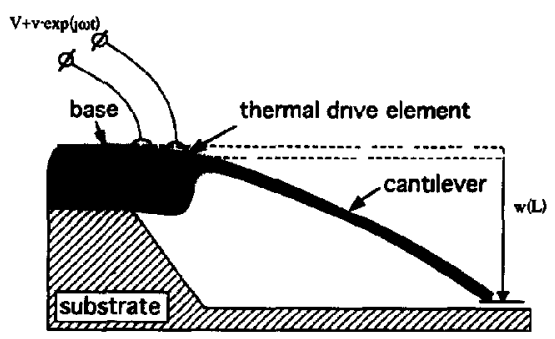

F1g 1 Sketch of cantllever with deep-diffused base with thermal excitation 
bend downward, a displacement and a rotation are imparted on the suspended edge of the compliant part of the structure Due to distributed inertial and damping forces, the latter will deform, and will vibrate with large amplitudes for frequencies around its natural fre quencies $[18,20]$ It is expected that for proper designs of the base and of the compliant part the resonance frequency of the structure will be determined by the material properties and dimensions of the single materal compliant thın part of the structure

Other authors have already pointed at advantages of a thick rim realized by deep boron diffusion, such as the relevant planar dimensions of the structure being determined by front side processes [21], and sharp corners with stress concentration being avoided [22,23] But for thermal excitation there are additional advantages in having the excitation at the thick base With the heat generation close to the support, and on a thick part of the structure, the thermal conductivity to the heat sink is expected to be large, and the resulting static temperature elevation, often a problem in thermal excitation [17], will be small Moreover, between limits, the efficiency of thermal excitation does not decrease with increasing thickness and decreasing length of the base If we assume a homogeneous base with heat generation located in the upper fibres, then the effective bending moment will have a magnitude $M_{\mathrm{e}}$ proportional to the flexural rigidity and independent of frequency for frequencies below a threshold frequency $v_{t}=2 a / h_{b}{ }^{2}$ [24], with $a$ the coefficient of thermal diffusivity of the base material and $h_{\mathrm{b}}$ the thickness of the base For silicon $a=62 \times 10^{-5} \mathrm{~m}^{2} / \mathrm{s}$, so that for $h_{\mathrm{b}}=15 \mu \mathrm{m}$ the threshold frequency equals $v_{\mathrm{t}}=550 \mathrm{kHz}$ The bending moment causes a rotation which is now independent of the thickness and length of the base, and is even independent of the length of the drive resistor' This means that for a $1 \mu \mathrm{m}$ thick 'base' the rotation would be the same, only the threshold frequency would be much larger'

Thermal base drive is attractive for several applications, such as resonant strain gauges, resonant proximity sensors, ultrasonic actuators and vibrating mirrors The resonance frequency is determined by the bare (simple and homogeneous) structure, and will therefore be more stable and more selectively sensitive than in the case of conventional drive Also, quality factors can be higher because of the absence of thin films of poor mechanical quality, and the absence of nearby external elements (squeeze film damping) Large forces or large displacements can bc obtaincd, although this strongly depends on the geometries of the compliant part of the structure and of the base, respectively This paper presents the realization of thermally excited cantilevers and microbndges suspended from deep boron diffused bases Models are derived for the thermal and mechan- ical behaviour of these structures, and these are verified by experiments

\section{Fabrication}

Test samples were fabricated from (100) slicon wafers, see Fig 2 Consecutive processing steps are

(1) thermal oxidation (wet atmosphere, $1100^{\circ} \mathrm{C}, 3 \mathrm{~h}$ )

(11) opening of diffusion windows (buffered HF)

(iii) double sided deep boron diffusion (solid source, $1150^{\circ} \mathrm{C}, 16 \mathrm{~h}$ )

(1v) opening of diffusion windows (buffered HF)

(v) shallow boron diffusion (solid source, $1150^{\circ} \mathrm{C}$, $40 \mathrm{~min}$ )

(vi) oxide strip (HF solution)

(vil) deposition of insulating layer (LPCVD silicon oxide)

(vii) opening of contact windows and of etch windows (buffered HF)

(1x) metallization (evaporation of chromum-gold, lift-off)

(x) bulk etch (EDP solution, $115^{\circ} \mathrm{C}, 1 \mathrm{~h}$ )

The contours of the diffusion regions sketched in Fig 2 are the expected positions of the boron concentration required for the etch stop $\left(5 \times 10^{+19} \mathrm{~cm}^{-3}\right.$ [25]) In the area where the shallow and deep diffusion regions overlap, superposition of the two profiles results in a smoothing of the aforementioned contour This smoothing effect will also occur where the thick base and the thick rim meet Exposed sharp outer edges of the diffusion profile such as at the tip of the cantilever and the edge of the nm are smoothed during the etching of the bulk

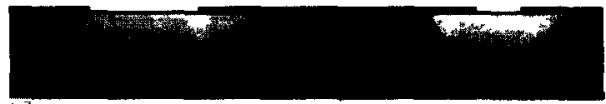

(a)

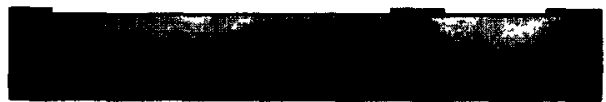

(b)

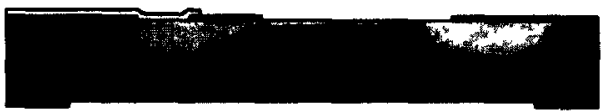

(c)

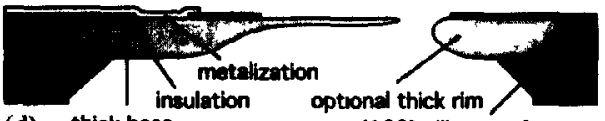

(d) thick base

(100) silicon wafer

Fig 2 States in the fabrication sequence after deep boron diffusion (a), after shallow boron diffusion (b), after metallization (c), after bulk etch (d) 


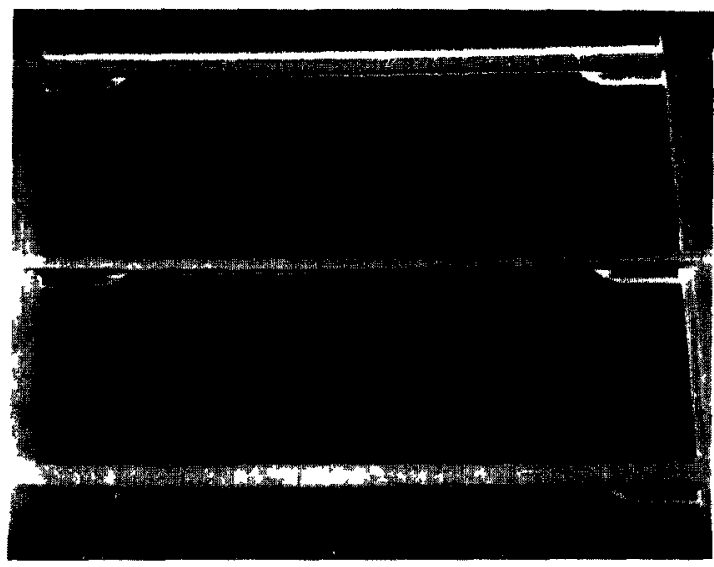

(a)

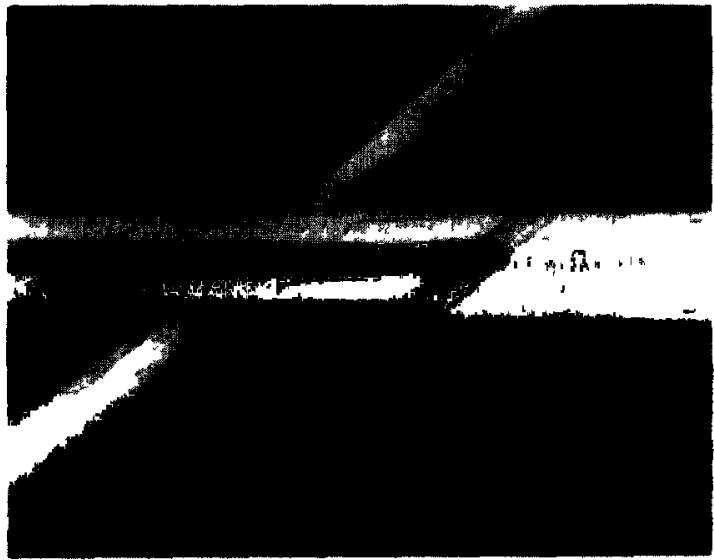

(b)

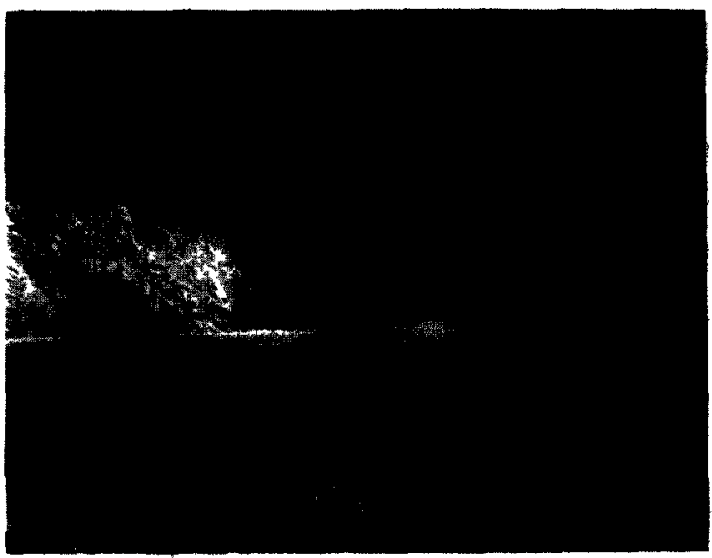

(c)

Fig 3 SEM photographs of realized structures (a) array of microbndges suspended by thick bases, (b) close-up of a thick base, (c) close-up of the backside of a thick base
F1gure 3 shows SEM photographs of realized structures Figure 3(a) shows an array of structures with $32 \mu \mathrm{m}$ thick and $293 \mu \mathrm{m}$ microbridges suspended by $15 \mu \mathrm{m}$ thick bases $52 \mu \mathrm{m}$ long Figure 3(b) shows a close-up of the top side of a $15 \mu \mathrm{m}$ thick base of length $25 \mu \mathrm{m}$ It also shows the dielectnc layer with contact holes and metallization Note the thick rum from which the base is suspended This photograph clearly shows the rounded shapes obtained with the diffusion based etch stop layer Figure 3(c) shows a close-up of the bottom side of a thick base of length $55 \mu \mathrm{m}$ It also shows the thick rim around the periphery of the etch hole This thick rum determines the lateral dimensions of the etched hole, and therefore allows for large differences in wafer thickness

\section{Cantilevers}

\section{Model}

For excitation of the cantilevers, a voltage $V+v \exp (j \omega)$ is applied across the resistive drive element with resistance $R$, leading to a dynamic power dissipation with a component of magnitude $P_{\mathrm{e}}=2 \mathrm{Vv} / R$ at the excitation frequency [14] This leads to an effective bending moment with magnitude $M_{c}=\alpha E I_{\mathrm{b}} P_{\mathrm{e}} /\left(\lambda A_{\mathrm{R}}\right)$ for frequencies below the threshold frequency $v_{\mathrm{t}}=2 a / h_{\mathrm{b}}{ }^{2}$ [24], with $\alpha$ the linear thermal expansion coefficient of the base material, $E I_{\mathrm{b}}$ the flexural rigidity of the base, $\lambda$ the coefficient of thermal conductivity, $A_{\mathrm{R}}$ the area of the resistor (width times length), $a$ the thermal diffusivity and $h_{\mathrm{b}}$ the thickness of the base material (we assume that the dissipation is concentrated in the upper fibres of the cross section) The bending moment $M_{\mathrm{e}}$ causes a rotation $\phi_{\mathrm{e}}=6 \alpha P_{\mathrm{e}} /\left(\lambda b_{\mathrm{b}}\right)$, with $b_{\mathrm{b}}$ the width of the base This leads to a nominal rotation $w^{\prime}(0)=\phi_{\mathrm{e}}$ and a defiection $w(0)=\phi_{c} L_{c}$ at the end of the base, with $L_{c}$ the distance between the centre of the drive element and the end of the base This causes a forced vibrational motion $w(x) \exp (j \omega t)$ of the ficxural cantilcver of length $L$, flexural rigidity $E I$, mass per unit length $\rho A$ and drag force per unit length $c$, with $x$ the distance to the edge of the drive element Distributed inertial and damping forces $\rho A w(x)+c w(x)$ act as a distributed transverse load on the cantilever An expression for $w(x)$ of the resulting vibration can be derived from [20]

$$
\begin{aligned}
w(x)= & w(0)\left(1+\sum_{i=1}^{\infty} \alpha_{i} X_{i}(x)\left(H_{i}(j \omega)-1\right)\right) \\
& +w^{\prime}(0)\left(x+\sum_{i=1}^{\infty} \beta_{i} X_{t}(x)\left(H_{i}(j \omega)-1\right)\right)
\end{aligned}
$$

where $X_{1}(x)$ is the shape function of the $t$ th mode for $0 \leqslant x \leqslant L\left(X_{1}(x)=0\right.$ for $\left.x \leqslant 0\right)$

$$
\begin{aligned}
X_{i}(x)= & \cosh \left(k_{i} x / L\right)-\cos \left(k_{1} x / L\right) \\
& -\sigma_{i}\left(\sinh \left(k_{2} x / L\right)-\sin \left(k_{1} x / L\right)\right)
\end{aligned}
$$


with $k_{\imath}$ the $\imath$ th root of $1+\cosh \left(k_{\imath}\right) \cos \left(k_{\imath}\right)=0$,

$\sigma_{\imath}=\left(\cosh \left(k_{t}\right)+\cos \left(k_{t}\right)\right) /\left(\sinh \left(k_{t}\right)+\sin \left(k_{t}\right)\right)$

$\alpha_{i}=\int X_{\mathrm{r}} \mathrm{d} x / L=2 \sigma_{\imath} / k_{t}$ and $\beta_{t}=\int X_{t} x \mathrm{~d} x / L^{2}=2 / k_{t}$

so that $k_{1}=1875, \sigma_{1}=0734, \alpha_{1}=0783, \beta_{1}=0569$ and $X_{2}(L)=2 \quad H_{2}(j \omega)$ 1s the second order transfer function $\quad H_{1}(j \omega)=1 /\left(1+\left(j \omega / \omega_{1}\right) / Q_{1}+\left(j \omega / \omega_{\imath}\right)^{2}\right)$, with $Q_{1}$ the quality factor of the $t$ th mode For practıcal values of $Q_{1}$ only one term needs to be considered near the $t$ th resonance frequency of the cantilever $v_{i}=1 /(2 \pi) k_{i}^{2} \sqrt{((E I) /(\mu A)) / L^{2}}$

The vibration of the cantilever causes a transverse reaction force and a bending moment exerted on the base The reaction loads are found from integration of the distributed inertial and damping forces over the length of the cantilever This gives an additional deformation of the base superimposed on the forced curvature due to the driving moment Figure 4(a) shows the predicted results for the modulus of the normalized tip defiection $\left|w(L) /\left(\phi_{\mathrm{e}} L\right)\right|$ versus normalized resonance frequency $v / v_{1}$ This particular plot was generated with a value $Q_{1}=100$ The plot shows a maximum value of magnitude $\left|w(L) /\left(\phi_{\mathrm{e}} L\right)\right|_{\max } \approx 2\left(\beta_{1}+\left(L_{\mathrm{e}} / L\right) \alpha_{1}\right) Q_{1}$, at nearly the resonance frequency $v \approx v_{1}$, and a bandwidth

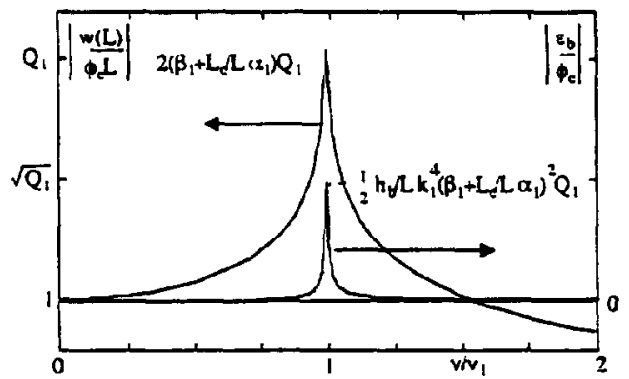

(a)

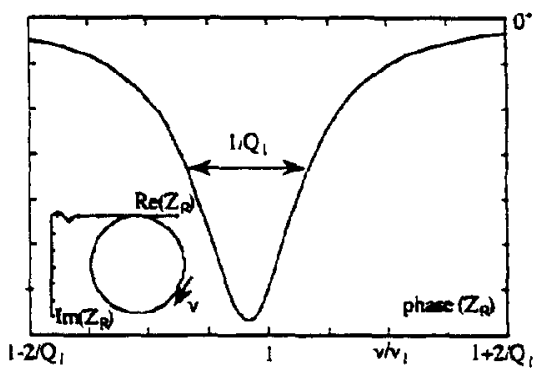

(b)

Fig 4 Predicted tip displacement $|w(L)|$ and bending strain at the drive element due to vibrational load $\left|\varepsilon_{\mathrm{b}}\right|$ related to nominal rotation $\left|\phi_{\mathrm{a}}\right|(\mathrm{a})$, and results of phase $\varphi$ of impedance $Z_{R}$ of piezoresistive dnve element (b), vs normalized resonance frequency $v / v_{1}$, with $v_{1}$ the fundamental resonance frequency of the 1deally clamped, undamped, cantilever of $v_{1} / Q_{1}$ at the $-3 \mathrm{~dB}$ level Also shown is the simulated result of the bending strain $\varepsilon_{\mathrm{b}}$ in the upper fibres at the centre of the drive element due to the vibrational load, divided by the nominal rotation $\phi_{0}$ The maximum value equals $\left|\varepsilon_{\mathrm{b}} / \phi_{\mathrm{e}}\right|_{\max } \approx(1 / 2)\left(h_{\mathrm{b}} / L\right) k_{1}{ }^{4}\left(\beta_{1}+\left(L_{\mathrm{e}} / L\right) \boldsymbol{x}_{1}\right)^{2} Q_{1}$

The vibration induced strain causes a change in the resistivity of drive resistor thanks to the piezoresistive effect Figure 4(b) shows a Bode plot of the phase of the impedance of the piezoresistive driving element For frequencies far away from resonance the impedance is equal to the resistance $R$ As a result of the deformation of the drive element due to the vibrational load, a loop emerges The resulting maximum phase shift equals

$$
\begin{aligned}
& \Delta \varphi_{\max } \approx\left(180^{\circ} / \pi\right) G 6 \alpha\left(2 V^{2} / R\right) /\left(\lambda b_{\mathrm{b}}\right) \\
&(1 / 2)\left(h_{\mathrm{b}} / L\right) k_{1}{ }^{4}\left(\beta_{1}+\left(L_{\mathrm{e}} / L\right) \alpha_{1}\right)^{2} Q_{1}
\end{aligned}
$$

where $G$ is the gauge factor of the resistor material For structures with a relatively compliant base decreases are found for the resonance frequency, tip deflection and phase shift of the impedance, with relative magnitudes of $3(E I / L) /\left(E I_{\mathrm{b}} / L_{\mathrm{b}}\right), 333(E I / L) /\left(E I_{\mathrm{b}} / L_{\mathrm{b}}\right)$ and $8(E I / L) /$ $\left(E I_{\mathrm{b}} / L_{\mathrm{b}}\right)$, respectively

\section{Experments}

Transfer functions and impedances were measured using an HP $4194 \mathrm{~A}$ network analyzer The displacement of the cantilever was monitored using a Michelson interferometer Figure 5(a) shows a Bode plot of the measured transfer function of the displacement of the tip of a thermally excitated cantilever ( \# C1) of length $800 \mu \mathrm{m}$ and thickness $3 \mu \mathrm{m}$, suspended by a base of length $100 \mu \mathrm{m}$, width $100 \mu \mathrm{m}$ and thickness $15 \mu \mathrm{m}$, and distance between resistor and the end of the base $L_{\mathrm{e}}=35 \mu \mathrm{m}$ The driving power required to get an amplitude of vibration equal to one fringe (approx $80 \mathrm{~nm}$ ) was determined It turned out that the structures have a built-in $\mathrm{dc}$ offset of approx $5 \mathrm{mV}$ Impedance measurements, as well as single-element two-port approach measurements [26], farled to show any response, even under vacuum conditions and with $13 \mathrm{~V} \mathrm{~d} \mathrm{c}$ voltage Therefore, to test the capability of piezoresistive detection, experiments were performed with acoustic excitation using a loudspeaker, and keeping the amplitude of vibration of the tip of the cantilever at resonance at one or more fringes The on-chip resistor was connected in a balanced Wheatstone-bridge Figure $5(\mathrm{~b})$ shows a Bode plot of the bridge output for a similar sample (\#C2) of length of $400 \mu \mathrm{m}$ in acoustic excitation Table 1 summarizes the results obtained from these measurements

In general the experimental results agree reasonably well with the predicted values The efficiencies of excitation and detection are higher than predicted This is remarkable because in the model it was assumed that 

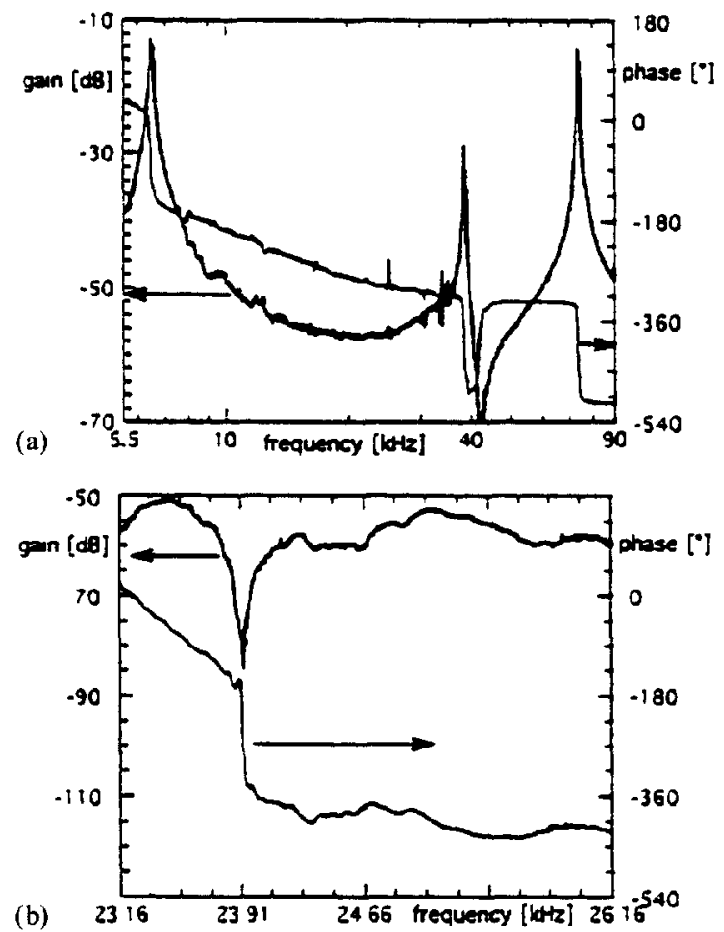

Fig 5 Experimental results Bode plots of tup displacement of sample \# $\mathrm{Cl}$ in thermal excitation (a), and of piezoresistive detection of sample \# $\mathrm{C} 2$ in acoustic excitation (b)

the electrical current is located in the upper fibres of the cross section of the drive element, while in practice the current flow is probably more three-dimensional The resonance frequencies found in acoustic excitation were a few percent lower than observed in experiments with thermal excitation In thermal excitation the resonance frequency was very stable, and hardly dependent on $d c$ power

\section{Microbridges}

Still the static part of the dissipation is expected to cause a static temperature elevation of the structure This temperature elevation is expected to be much smaller for thermal excitation at a thick base compared to conventional thermal excitation Experiments were carned out on microbridges of lengths $893 \mu \mathrm{m}$, suspended by two identical thick bases of the latter the distance $L_{\mathrm{e}}$ between the centre of the resistor and the end of the base was constant, while the distance $L_{\mathrm{Q}}$ between the resistor and the support (a thick rum) was varied Detection of the vibration at the centre of the microbridge was achieved using a Michelson interferometer An HP 4194 A network analyzer was used to measure the frequency response From this the frequency at the first resonance mode was determined For each sample the driving voltages $V$ and $v$ were varied This results in varying static dissipations $Q=\left(V^{2}+05 v^{2}\right) / R$ [14] Figure 6(a) shows the measured resonance frequency versus static heat $Q$ The influence of the static heat generation on the resonance frequency stems from the temperature elevation of the structure due to the heat generation The temperature elevation is expected to be smaller for smaller distances $L_{\mathrm{Q}}$ of the heat source to the heat sink The temperature elevation leads to a thermal expansion of the thin microbridge as well as of the thick base This expansion causes a compressive axial force in the structure This force is supenmposed on the initial tensile axial force inherent with boron doping, and hence causes a shift of the resonance frequency of the structure For a large range of axial forces $N$, the second power of the resonance frequency is linear with the axial force [27]

$$
\Delta\left(v_{\mathrm{res}}^{2}\right)=-0312 \frac{E}{\rho} \frac{1}{L^{2}} \frac{N}{E A}
$$

TABLE 1 Experımental and predicted results for cantilevers with thermal excitation at deep-diffusion base

\begin{tabular}{lll}
\hline & Sample & \\
\cline { 2 - 3 } & $\# \mathrm{C} 1$ & \#C2 \\
\hline Length $(\mu \mathrm{m})$ & 800 & 400 \\
Resistance drive element $(\Omega)$ & 4 & 4 \\
Fund resonance frequency $(\mathrm{kHz})$ & $(582)$ & $(233)$ \\
$\quad$ in thermal excitation & 6504 & 2466 \\
in acoustic excitation & 6334 & 2391 \\
Quality factor & 62 & 138 \\
Tip displaccment at resonancc $(\mathrm{nm} / \mathrm{mW})$ & $160(57)$ & $30(63)$ \\
Bending strain drive element & $\Delta R / R=2 \times 10^{-3}$ & $\Delta R / R=8 \times 10^{-3}$ \\
at 80 nm tip displacement & $\left(\varepsilon_{\mathrm{b}}=35 \times 10^{-6}\right)$ & $\left(\varepsilon_{\mathrm{b}}=14 \times 10^{-5}\right)$ \\
Maximum phase shift, $Z_{\mathrm{p}}(\mathrm{mdeg})$ & $-(01)$ & $-(06)$ \\
\hline
\end{tabular}

Base thickness $h_{\mathrm{b}}=15 \mu \mathrm{m}$, length $L_{\mathrm{b}}=100 \mu \mathrm{m}$, width $b_{\mathrm{b}}=100 \mu \mathrm{m}$, distance between resistor and end of base $L_{\mathrm{c}}=35 \mu \mathrm{m}$ Cantilever thickness $h=3 \mu \mathrm{m}$, mass density $\rho=233 \times 10^{3} \mathrm{~kg} / \mathrm{m}^{3}$, Young's modulus $E=17 \times 10^{11} \mathrm{~N} / \mathrm{m}^{2}$ Predicted values in parentheses 


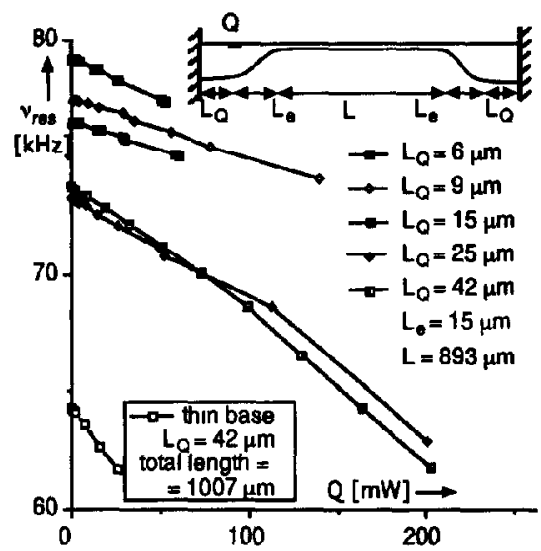

(a)

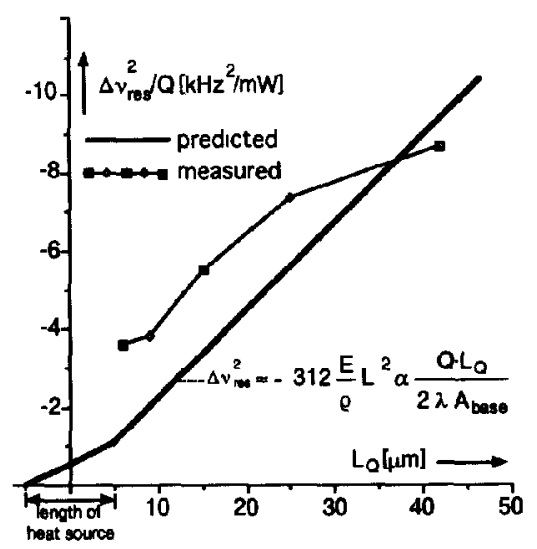

(b)

Fig 6 Measured resonance frequencies $v_{\text {res }}$ vs static heat generation $Q$ for different distances $L_{Q}$ of heater to support (a) and measured and predicted $\Delta\left(v_{\text {res }}{ }^{2}\right) / Q$ vs $L_{Q}$ (b)

Figure 6(b) shows a plot of the predicted and measured frequency shift per $\mathrm{mW}$ heat generation versus distance $L_{\mathrm{Q}}$ The measured curve shows the same trend as the predicted curve, only the influence of $L_{Q}$ is smaller than predicted Also, for small values of $L_{\mathrm{Q}}$ the thermal conductivity to the ideal heat sink appears to be larger than predicted Both discrepancies are probably caused by the influence of the thick rim In order to obtain varying distances $L_{\mathrm{Q}}$ we simply varied the width of the rim in our mask layout Hence, a smaller value of $L_{\mathrm{Q}}$ is accompanied by a larger thermal resistance of the thick rim The latter was not accounted for in our model For structures without the thick rim, the frequency shifts are expected to be smaller For larger values of $L_{\mathrm{Q}}$ the measured curve drops below the predicted curve One possible explanation might be that heat transfer directly to the surrounding air plays a role However, we think that this decrease of the slope stems from the effect of a small initial deflection of the microbridge, which leads to a smaller impact of the thermal expansion than expected according to eqn (2) [27]

\section{Discussion}

The resonance frequencies are almost exclusively determined by the fiexural structures The tip displacement is almost independent of the stiffness of the base Magnitudes in the order of $1 \mu \mathrm{m}$ are easily obtained Detection of the vibration at the base has a low sensitivity, proportional to the ratio of stiffnesses of the cantilever and of the base Even so, strains at the base in the order of $10^{-5}$ are easily obtained, which could be sufficient

To get any excitation, the drive element should have a finite stiffness The efficiency of detection at the base is even proportional to the ratio of stiffness of the cantilever over stuffness of the drive element But if the drive element is too compliant it becomes part of the flexural structure, and it will affect the device characteristics such as the resonance frequency and tip displacement Therefore, we should define 'base drive' as the drive with negligible effect on device characteristics In this sense the excitation applied by Brennen et al [7] is 'base drive', while that applied by Moser et al [16] is not

'Base drive' is surtable both for resonant sensors as well as for actuators The resonance frequency is determined by a bare (simple and homogeneous) structure, and will therefore be more stable and more selectively sensitive than in the case of conventional drive Also, quality factors can be higher because of the absence of thun films of poor mechanceal quality, and the absence of nearby external elements (squeeze film damping) Large forces or large displacements can be obtained, although this strongly depends on the geometry of the flexural cantilever and of the base, respectively

The fabrication of the devices is simple and requires only 5 masks, or even 4 masks if bulk etching is done from the front side [18] The forced rotation of the base is independent of the thickness and the length of the excitation area Therefore the carrier of the thermal excitation element may as well be thick and the resistor may as well be close to the substrate This way the thermal conductance to the substrate is large, so that the temperature elevation of the structure, often a drawback of thermal excitation [17], will be small Alternatively, a relatively large excitation power can be applied without thermal breakdown of the resistor This makes thermal excitation at a thick base useful even for actuators for which a large amplitude of vibration is required

The efficiency of thermal excitation and piezoresistive detection will probably be higher if thin film resistors on top of the base are used, rather than having dissipa- 
tion in the bulk of the $\mathrm{p}^{++}$base as we have done in the investigated samples Current research focusses on samples with polysilicon resistors

\section{Conclusions}

The novel approach of thermal excitation employing deep-diffusion bases was presented Device characteristics such as resonance frequencies and amplitude of vibration can be independent of the geometry of the drive element, while the efficiency of detection at the base is proportional to the ratio of stiffnesses of the compliant structure and the drive element Static temperature elevations are smaller than for conventional structures with thermal excitation with equivalent power dissipations Larger voltages and currents can be allowed without disturbance of device performance $A$ tip displacement of $1 \mu \mathrm{m}$ and a detection strain of $10^{-5}$ are easily obtained

\section{Acknowledgements}

The authors thank the staff of the clean room of the MESA research institute for their assistance Fabrication was performed under grant \# ECS-8915215 from the US National Science Foundation The research of Dr Bouwstra has been made possible by a fellowship of the Royal Netherlands Academy of Sciences

\section{References}

$1 \mathrm{H}$ A C Tilmans, $M$ Elwenspoek and $\mathrm{J} \mathrm{H} \mathrm{J}$ Fluitman, Micro-resonant force gauges, Sensors and Actuators $A, 30$ (1992) 35-53

2 G Sternme, Resonant silicon sensors, $J$ Micromech Microeng , 1 (1991) 113-125

3 A P Pisano, Resonant-structure micromotors histoncal perspectıve and analysis, Sensors and Actuators A, 20 (1989) $83-89$

4 H C Nathanson, W E Newell, R A Wickstrom and J R Davis, $\mathrm{Jr}$, The resonant gate transistor, IEEE Trans Electron Devices, ED-14 (1967) 117-133

5 R T Howe and R S Muller, Resonant-microbridge vapor sensor, IEEE Trans Electron Devices, ED-33 (1986) 499-506

6 W C Tang, T-C H Nguyen and R T Howe, Laterally driven polysilicon resonant microstructures, Sensors and Actuators $A$, 20 (1989) 25-32

7 R A Brennen, A P Pisano and W C Tang, Multiple mode micromechanical resonators, Proc MEMS-90, Napa Valley, $C A, U S A, 1990$, pp 9-14

$8 \mathrm{~K}$ Ikeda, H Kuwayama, $\mathrm{T}$ Kobayashı, $\mathrm{T}$ Watanabe, $\mathrm{T}$ Nishıkawa, T Yoshıda and $\mathbf{K}$ Harada, Silıcon pressure sensor integrates resonant strain gauge on draphragm, Sensors and Actiators A, $21-23$ (1990) 146-150
9 B Wagner and W Benecke, Microfabricated actuator with moving permanent magnet, Proc MEMS-91, Nara, Japan, 1991, pp 27-32

$10 \mathrm{~W} C$ Blanchard, Design of a resonant pressure sensor, $I n$ strum Control Syst, (1972) 35-36

$11 \mathrm{~J} G$ Smits, $H$ A C Tilmans and $T$ S J Lammerink, Frequency dependence of resonant diaphragm pressure sensor, Proc Transducers' 85, Philadelphia, PA, USA, 1985, pp 93-96

$12 \mathrm{~F} \mathbf{R}$ Blom, S Bouwstra, J H J Fluitman and M Elwenspoek, Resonating silicon beam force sensor, Sensors and Actuators, 17 (1990) 513-519

$13 \mathrm{R}$ J Wilfinger, P H Bardell and D S Chhabra, The resonistor a frequency selective device utilizing the mechanical resonance of a slicon substrate, IBM $J$ Res Dev, 12 (1968) $113-118$

14 T S J Lammerınk and W Wlodarsk, Integrated thermally excited resonant diaphragm pressure sensor, Proc Transducers 85, Phlladelphia, PA, USA, 1985, pp 97-100

15 S Bouwstra, R Legtenberg, H A C Tilmans and M Elwenspoek, Resonating microbridge mass flow sensor, Sensors and Actuators A, 2I-23 (1990) 332-335

$16 \mathrm{D}$ Moser, $\mathrm{O}$ Brand and $\mathrm{H}$ Baltes, A CMOS-compatıble thermally excited silicon oxide beam resonator with aluminum mirror, Proc Transducers '91, San Francisco, CA, USA, 1991, pp $547-550$

17 T S J Lammerınk, M Elwenspoek, R H van Ouwerkerk, S Bouwstra and $\mathrm{J} \mathrm{H} \mathrm{J} \mathrm{Flutman,} \mathrm{Performance} \mathrm{of} \mathrm{thermally}$ excited resonators, Sensors and Actuators A, 21-23 (1990) $352-356$

18 S Bouwstra, H A C Tilmans, A Selvakumar and K Najafi, Base-driven micromechanical resonators, Proc IEEE Workshop Solld-State Sensors and Actuators, Hilton Head, SC, USA, June 1992, pp 148-152

$19 \mathrm{~K}$ Najafi and $\mathrm{K} \mathrm{D}$ Wise, High yicld IC-compatible multichannel recording array, IEEE Electron Devices Meet, EDM32 (1985) 1206-1211

20 S Timoshenko, D H Young and W Weaver, Jr, Vibration Problems in Engineering, Wiley, New York, 1974, pp 443-447

21 S T Cho, K Najafi and K D Wise, Intrinsic stress compensation and scaling in ultrasensitive silicon pressure sensors, IEEE Electron Devices, ED-39 (1992) 836-842

$22 \mathrm{~J} F$ Hetke, $\mathrm{K}$ Najafi and $\mathrm{K}$ D Wise, Flexible silicon interconnects for microelectromechanical systems, Proc Transducers '91, San Francisco, CA, USA, 1991, pp 764767

23 F Pourahmad, D Gee and K Petersen, The effect of corner radius of curvature on the mechanical strength of micromachined single-crystal silicon structures, Proc Transducers '91, San Francisco, CA, USA, 1991, pp 197-200

24 T S J Lammerınk, M Elwenspoek and J H J Fluitman, Frequency dependence of thermal excitation of micromechantcal resonators, Sensors and Actuators A, 25-27 (1991) 685689

25 J C Greenwood, Ethylene diamıne-catechol-water muxture shows preferential etching of $\mathrm{p}-\mathrm{n}$ junction, $J$ Electrochem Soc, 116 (1969) 1326-1327

26 H A C Tilmans, D J Ijntema and J H J Fluitman, Single element excitation and detection of (micro-)mechanical resonators, Proc Transducers' 91, San Francisco, CA, USA, 1991, pp 533-537

$27 \mathrm{~S}$ Bouwstra and $\mathbf{H} \mathbf{J}$ Geljselaers, On the resonance frequencies of microbridges, Proc Transducers' 91 , San Francisco, $C A$, USA, 1991, pp 538-542 